Appl. Set-Valued Anal. Optim. 2 (2020), No. 2, pp. 205-222

Available online at http://asvao.biemdas.com

https://doi.org/10.23952/asvao.2.2020.2.06

\title{
STRONG CONVERGENCE THEOREMS OF A SPLIT COMMON NULL POINT PROBLEM AND A FIXED POINT PROBLEM IN HILBERT SPACES
}

\author{
MINH TUYEN TRUONG ${ }^{1, *}$, THI THU THUY NGUYEN ${ }^{2}$, MINH TRANG NGUYEN $^{3}$ \\ ${ }^{1}$ Department of Mathematics and Informatics, Thainguyen University of Sciences, Thai Nguyen, Vietnam \\ ${ }^{2}$ School of Applied Mathematics and Informatics, Hanoi University of Science and Technology, Hanoi, Vietnam \\ ${ }^{3}$ Faculty of International Training, Thainguyen University of Technology, Thai Nguyen, Vietnam
}

\begin{abstract}
In this paper, we introduce a new iterative method for finding a common solution of the split common null point problem and the fixed point problem in Hilbert spaces. We obtain the strong convergence of the new iterative method. We also give some applications and numerical experiments to support our main convergence results.
\end{abstract}

Keywords. Split common null point problem; Monotone operator; Metric projection; Nonexpansive mapping.

\section{INTRODUCTION}

Let $C$ and $Q$ be nonempty closed and convex subsets of real Hilbert spaces $H_{1}$ and $H_{2}$, respectively. Let $T: H_{1} \rightarrow H_{2}$ be a bounded linear operator and let $T^{*}: H_{2} \rightarrow H_{1}$ be the adjoint of $T$. The split feasibility problem (SFP) is formulated as follows:

$$
\text { find an element } \quad x^{*} \in S=C \cap T^{-1}(Q)
$$

(1.1), which was first introduced by Censor and Elfving [5] for modeling inverse problems, plays an important role in the real world, such as, in medical image reconstruction and signal processing [2, 3, 4]. Recently, (1.1) has been under the spotlight of research. For recent celebrated results, we refer to $[4,5,15,22,23,28,29]$ and the references therein.

A special case of (1.1) is called the convex constrained linear inverse problem [7], which consists of finding an element $x^{*} \in H_{1}$ such that

$$
x^{*} \in C \text { and } T x^{*}=b \in Q .
$$

If $C$ is a closed convex subset of a Hilbert space $H$, then $C$ is the set of null points of the maximal monotone operator $A$, which is defined by $A=\partial i_{C}$, where $i_{C}$ is the indicator function of $C$ and $\partial i_{C}$ is the subdifferential operator of $i_{C}$. So, (1.1) becomes a special case of the split common null point problem (SCNPP), which consists of finding a point $x^{*} \in H_{1}$ such that

$$
0 \in A_{1}\left(x^{*}\right) \text { and } 0 \in A_{2}\left(T x^{*}\right),
$$

\footnotetext{
${ }^{*}$ Corresponding author.

E-mail addresses: tuyentm@tnus.edu.vn (M.T. Truong), thuy.nguyenthithu2@ hust.edu.vn (T.T.T. Nguyen), nguyenminhtrang@tnut.edu.vn (M.T. Nguyen).

Received April 24, 2020; Accepted May 31, 2020.
}

(C)2020 Applied Set-Valued Analysis and Optimization 
where $A_{i}: H_{i} \rightarrow 2^{H_{i}}, i=1,2$ are maximal monotone operators. This problem has been studied by many authors; see, e.g., [6, 9, 10, 24, 25, 27].

In 2008, Moudafi [13] introduced and studied the following Mann-type iterative method [12] for finding a common element of the set of solutions of a mixed equilibrium problem and the set of fixed points of a nonexpansive mapping:

$$
\begin{aligned}
& x_{0}=x \in C, \\
& y_{n}=T_{r_{n}}^{F}\left(x_{n}-r_{n} f x_{n}\right), \\
& x_{n+1}=\beta_{n} x_{n}+\left(1-\beta_{n}\right) S y_{n},
\end{aligned}
$$

where $\left\{r_{n}\right\} \subset(0, \infty),\left\{\beta_{n}\right\} \subset(0,1), F: C \times C \rightarrow \mathbb{R}$ is a bifunction, $C$ is a closed and convex subset of $H_{1}, S: C \rightarrow C$ is a nonexpansive mapping and

$$
T_{r}^{F} x=\left\{z \in C: F(z, y)+\frac{1}{r}\langle y-z, z-x\rangle \geq 0 \forall y \in C\right\} .
$$

Recently, Takahashi and Takahashi [20] extended Moudafi's result in another direction by combining the Mann iterative method and the Halpern iterative method [8] as follows:

$$
\begin{aligned}
& x_{0}=x \in C, u \in C, \\
& y_{n}=T_{r_{n}}^{F}\left(x_{n}-r_{n} f x_{n}\right), \\
& x_{n+1}=\beta_{n} x_{n}+\left(1-\beta_{n}\right) S\left[\alpha_{n} u+\left(1-\alpha_{n}\right) y_{n}\right] .
\end{aligned}
$$

Under some suitable conditions on the sequences $\left\{r_{n}\right\},\left\{\beta_{n}\right\}$, and $\left\{\alpha_{n}\right\}$, they proved the strong convergence of the sequence $\left\{x_{n}\right\}$ generated by the above iterative method.

Motivated by the work of Moudafi [13] and Takahashi and Takahashi [20], we introduce a iterative methods based on the Mann iterative method and the Halpern/viscosity iterative method for finding a common solution of the split common null point problem and the fixed point problem of a nonexpansive mapping. The split minimum point problem, the split feasibility problem, the split equilibrium problem, and the split variational inequality problem are also investigated as the applications of our main results. Finally, two numerical examples are also given to illustrate the effectiveness of the proposed algorithms.

\section{PRELIMINARIES}

Let $C$ be a nonempty, closed and convex subset of a Hilbert space $H$. For each $x \in H$, there is a unique $P_{C}^{H} x \in C$ such that

$$
\left\|x-P_{C}^{H} x\right\|=\inf _{u \in C}\|x-u\|
$$

and the mapping $P_{C}^{H}: H \rightarrow C$ defined by (2.1) is called the metric projection from $H$ onto $C$. Moreover, we have

$$
\left\langle x-P_{C}^{H} x, y-P_{C}^{H} x\right\rangle \leq 0, \forall x \in H, y \in C .
$$

Recall that a mapping $S: C \rightarrow C$ is said to be nonexpansive mapping if

$$
\|S x-S y\| \leq\|x-y\|, \quad \forall x, y \in C .
$$

We denote by $\operatorname{Fix}(S)$ the set of fixed points of $S$, i.e., $\operatorname{Fix}(S)=\{x \in C: S x=x\}$. 
For an operator $A: H \rightarrow 2^{H}$, we define its domain, range, and graph as follows:

$$
\begin{aligned}
& D(A)=\{x \in H: A(x) \neq \emptyset\}, \\
& R(A)=\cup\{A z: z \in D(A)\},
\end{aligned}
$$

and

$$
G(A)=\{(x, y) \in H \times H: x \in D(A), y \in A(x)\},
$$

respectively. The inverse $A^{-1}$ of $A$ is defined by

$$
x \in A^{-1}(y) \text { if and only if } y \in A(x) .
$$

The operator $A$ is said to be monotone if, for each $x, y \in D(A),\langle u-v, x-y\rangle \geq 0$ for all $u \in A(x)$ and $v \in A(y)$. We denote by $I^{H}$ the identity operator on $H$. A monotone operator $A$ is said to be maximal monotone if there is no proper monotone extension of $A$ or $R\left(I^{H}+\lambda A\right)=H$ for all $\lambda>0$. If $A$ is monotone, then we can define, for each $\lambda>0$, a nonexpansive single-valued mapping $J_{\lambda}^{A}: R\left(I^{H}+\lambda A\right) \rightarrow D(A)$ by

$$
J_{\lambda}^{A}=\left(I^{H}+\lambda A\right)^{-1}
$$

which is called the resolvent of $A$.

A monotone operator $A$ is said to satisfy the range condition if $\overline{D(A)} \subset R\left(I^{H}+\lambda A\right)$ for all $\lambda>0$, where $\overline{D(A)}$ denotes the closure of the domain of $A$. For a monotone operator $A$, which satisfies the range condition, we have $A^{-1}(0)=\operatorname{Fix}\left(J_{\lambda}^{A}\right)$ for all $\lambda>0$. If $A$ is a maximal monotone operator, then $A$ satisfies the range condition.

The following lemmas will be needed in what follows for the proof of the main results in this paper.

Lemma 2.1. [1] Let $A: D(A) \subset H \rightarrow 2^{H}$ be a monotone operator. Then, for $\lambda, \mu>0$, and $x \in D(A)$, we have

$$
J_{\lambda}^{A} x=J_{\mu}^{A}\left(\frac{\mu}{\lambda} x+\left(1-\frac{\mu}{\lambda}\right) J_{\lambda}^{A} x\right)
$$

Lemma 2.2. Let $A: D(A) \subset H \rightarrow 2^{H}$ be a monotone operator. Then, we have the following statements:

i) for $r \geq s>0$, we have

$$
\left\|x-J_{s}^{A} x\right\| \leq 2\left\|x-J_{r}^{A} x\right\|,
$$

for all $x \in R\left(I^{H}+r A\right) \cap R\left(I^{H}+s A\right)$;

ii) for all $r>0$ and for all $x, y \in R\left(I^{H}+r A\right)$, we have

$$
\left\langle x-y, J_{r}^{A} x-J_{r}^{A} y\right\rangle \geq\left\|J_{r}^{A} x-J_{r}^{A} y\right\|^{2} ;
$$

iii) for all $r>0$ and for all $x, y \in R\left(I^{H}+r A\right)$, we have

$$
\left\langle\left(I^{H}-J_{r}^{A}\right) x-\left(I^{H}-J_{r}^{A}\right) y, x-y\right\rangle \geq\left\|\left(I^{H}-J_{r}^{A}\right) x-\left(I^{H}-J_{r}^{A}\right) y\right\|^{2} ;
$$

iv) if $\Omega=A^{-1}(0) \neq \emptyset$, then, for all $x^{*} \in \Omega$ and for all $x \in R\left(I^{H}+r A\right)$,

$$
\left\|J_{r}^{A} x-x^{*}\right\|^{2} \leq\left\|x-x^{*}\right\|^{2}-\left\|x-J_{r}^{A} x\right\|^{2} .
$$


Proof. i) From Lemma 2.1, we have

$$
\begin{aligned}
\left\|x-J_{s}^{A} x\right\| & \leq\left\|x-J_{r}^{A} x\right\|+\left\|J_{r}^{A} x-J_{s}^{A} x\right\| \\
& \leq\left\|x-J_{r}^{A} x\right\|+\left(1-\frac{s}{r}\right)\left\|x-J_{r}^{A} x\right\| \\
& \leq 2\left\|x-J_{r}^{A} x\right\| .
\end{aligned}
$$

ii) Let $u=J_{r}^{A} x$ and $v=J_{r}^{A} y$. From the definition of $J_{r}^{A}$, we have $x \in u+r A(u)$ and $y \in v+r A(v)$. Thus, it follows from the monotonicity of $A$ that

$$
\frac{1}{r}\langle u-v, x-u-(y-v)\rangle \geq 0 \text {. }
$$

So,

$$
\langle x-y, u-v\rangle \geq\|u-v\|^{2}
$$

that is,

$$
\left\langle x-y, J_{r}^{A} x-J_{r}^{A} y\right\rangle \geq\left\|J_{r}^{A} x-J_{r}^{A} y\right\|^{2} .
$$

iii) Note that

$$
\begin{aligned}
\left\langle\left(I^{H}-J_{r}^{A}\right) x-\left(I^{H}-J_{r}^{A}\right) y, x-y\right\rangle= & \left\|\left(I^{H}-J_{r}^{A}\right) x-\left(I^{H}-J_{r}^{A}\right) y\right\|^{2} \\
& +\left\langle\left(I^{H}-J_{r}^{A}\right) x-\left(I^{H}-J_{r}^{A}\right) y, J_{r}^{A} x-J_{r}^{A} y\right\rangle \\
= & \left\|\left(I^{H}-J_{r}^{A}\right) x-\left(I^{H}-J_{r}^{A}\right) y\right\|^{2} \\
& +\left\langle x-y, J_{r}^{A} x-J_{r}^{A} y\right\rangle-\left\|J_{r}^{A} x-J_{r}^{A} y\right\|^{2} .
\end{aligned}
$$

From ii), we get

$$
\left\langle\left(I^{H}-J_{r}^{A}\right) x-\left(I^{H}-J_{r}^{A}\right) y, x-y\right\rangle \geq\left\|\left(I^{H}-J_{r}^{A}\right) x-\left(I^{H}-J_{r}^{A}\right) y\right\|^{2} .
$$

iv) Since $x^{*} \in A^{-1}(0)$, we have $x^{*} \in \operatorname{Fix}\left(J_{r}^{A}\right)$. It follows from iv) that

$$
\begin{aligned}
\left\|J_{r}^{A} x-x^{*}\right\|^{2} & =\left\|x-x^{*}\right\|^{2}+\left\|x-J_{r}^{A} x\right\|^{2}+2\left\langle J_{r}^{A} x-x, x-x^{*}\right\rangle \\
& =\left\|x-x^{*}\right\|^{2}+\left\|x-J_{r}^{A} x\right\|^{2}-2\left\langle\left(I^{H}-J_{r}^{A}\right) x-\left(I^{H}-J_{r}^{A}\right) x^{*}, x-x^{*}\right\rangle \\
& \leq\left\|x-x^{*}\right\|^{2}-\left\|x-J_{r}^{A} x\right\|^{2} .
\end{aligned}
$$

This completes the proof.

Lemma 2.3. [30] Let $T$ be a nonexpansive self-mapping on a nonempty closed and convex subset $C$ of a Hilbert space $H$. If $T$ has a fixed point, then $I^{H}-T$ is demiclosed, that is, whenever $\left\{x_{n}\right\}$ is a sequence in $C$ weakly converging to some $x \in C$ and the sequence $\left\{\left(I^{H}-T\right)\left(x_{n}\right)\right\}$ strongly converges to some $y$, it follows that $\left(I^{H}-T\right)(x)=y$.

Lemma 2.4. [19] Let $\left\{x_{n}\right\}$ and $\left\{y_{n}\right\}$ be bounded sequences in a Hilbert space $H$ and $\left\{\beta_{n}\right\}$ be a sequence in $(0,1)$ with $0<\liminf _{n \rightarrow \infty} \beta_{n} \leq \limsup _{n \rightarrow \infty} \beta_{n}<1$. Let $x_{n+1}=\left(1-\beta_{n}\right) y_{n}+\beta_{n} x_{n}$ for all $n \geq 0$ and $\limsup _{n \rightarrow \infty}\left(\left\|y_{n+1}-y_{n}\right\|-\left\|x_{n+1}-x_{n}\right\|\right) \leq 0$. Then $\lim _{n \rightarrow \infty}\left\|x_{n}-y_{n}\right\|=0$.

Lemma 2.5. [11] Let $\left\{s_{n}\right\}$ be a sequence of nonnegative numbers, $\left\{\alpha_{n}\right\}$ be a sequence in $(0,1)$ and $\left\{c_{n}\right\}$ be a sequence of real numbers satisfying the conditions:

i) $s_{n+1} \leq\left(1-\alpha_{n}\right) s_{n}+\alpha_{n} c_{n}$

ii) $\sum_{n=0}^{\infty} \alpha_{n}=\infty, \limsup _{n \rightarrow \infty} c_{n} \leq 0$.

Then $\lim _{n \rightarrow \infty} s_{n}=0$. 


\section{MAIN RESULTS}

Let $H_{1}$ and $H_{2}$ be two real Hilbert spaces. Let $A: H_{1} \rightarrow 2^{H_{1}}$ and $B: H_{2} \rightarrow 2^{H_{2}}$ be two maximal monotone operators on $H_{1}$ and $H_{2}$, respectively. Let $S: H_{1} \rightarrow H_{1}$ be a nonexpansive mapping and let $T: H_{1} \rightarrow H_{2}$ be a bounded linear operator from $H_{1}$ onto $H_{2}$. Suppose that $\Omega=A^{-1} 0 \cap T^{-1}\left(B^{-1} 0\right) \cap \operatorname{Fix}(S) \neq \emptyset$. We consider the following problem:

$$
\text { find an element } x^{\dagger} \in \Omega \text {. }
$$

Next, we propose the main iterative method for solving Problem (3.1). For any $u, x_{0} \in H_{1}$, generate a sequence $\left\{x_{n}\right\}$ as follows

$$
\begin{aligned}
& y_{n}=J_{\gamma_{n}^{A}}^{A} x_{n}, \\
& z_{n}=J_{\gamma_{n}^{B}}^{B}\left(T y_{n}\right), \\
& t_{n}=y_{n}+\delta T^{*}\left(z_{n}-T y_{n}\right), \\
& x_{n+1}=\beta_{n} x_{n}+\left(1-\beta_{n}\right)\left[\alpha_{n} u+\left(1-\alpha_{n}\right) S t_{n}\right], n \geq 0,
\end{aligned}
$$

where $\left\{\gamma_{n}^{A}\right\},\left\{\gamma_{n}^{B}\right\},\left\{\beta_{n}\right\}$, and $\left\{\alpha_{n}\right\}$ are sequences of positive real numbers.

We are now in a position to prove the strong convergence of the sequence $\left\{x_{n}\right\}$ generated in (3.2) under the following conditions:

C1) $\min \left\{\inf _{n}\left\{\gamma_{n}^{A}\right\}, \inf _{n}\left\{\gamma_{n}^{B}\right\}\right\}=r>0, \lim _{n \rightarrow \infty}\left|\gamma_{n+1}^{A}-\gamma_{n}^{A}\right|=0$;

C2) $0<\liminf _{n \rightarrow \infty} \beta_{n} \leq \limsup _{n \rightarrow \infty} \beta_{n}<1$;

C3) $\sum_{n=1}^{\infty} \alpha_{n}=\infty$ and $\lim _{n \rightarrow \infty} \alpha_{n}=0$;

C4) $\delta \in\left(0, \frac{2}{\|T\|^{2}}\right)$.

Theorem 3.1. If the conditions $\mathrm{C} 1)-\mathrm{C} 4)$ are satisfied, then the sequence $\left\{x_{n}\right\}$ generated by (3.2) converges strongly to $x^{\dagger}=P_{\Omega}^{H_{1}} u$ as $n \rightarrow \infty$.

Proof. First, we prove that the sequence $\left\{x_{n}\right\}$ is bounded.

Fix $p \in \Omega$. From $p \in A^{-1} 0$ and Lemma 2.2 iv), we have

$$
\left\|y_{n}-p\right\|^{2} \leq\left\|x_{n}-p\right\|^{2}-\left\|x_{n}-J_{\gamma_{n}^{A}}^{A} x_{n}\right\|^{2} .
$$

From $T p=J_{\gamma_{n}^{B}}^{B}(T p)$ and Lemma $\left.2.2 \mathrm{iv}\right)$, we have

$$
\left\|z_{n}-T p\right\|^{2} \leq\left\|T y_{n}-T p\right\|^{2}-\left\|T y_{n}-J_{\gamma_{n}^{B}}^{B}\left(T y_{n}\right)\right\|^{2} .
$$

From Lemma 2.2 iii), we have

$$
\begin{aligned}
\left\|t_{n}-p\right\|^{2} & =\left\|y_{n}-p\right\|^{2}+\delta^{2}\left\|T^{*}\left(z_{n}-T y_{n}\right)\right\|^{2}+2 \delta\left\langle y_{n}-p, T^{*}\left(z_{n}-T y_{n}\right)\right\rangle \\
& \leq\left\|y_{n}-p\right\|^{2}-\delta\left(2-\delta\|T\|^{2}\right)\left\|z_{n}-T y_{n}\right\|^{2} \\
& \leq\left\|x_{n}-p\right\|^{2}-\left\|x_{n}-J_{\gamma_{n}^{A}}^{A} x_{n}\right\|^{2}-\delta\left(2-\delta\|T\|^{2}\right)\left\|z_{n}-T y_{n}\right\|^{2} .
\end{aligned}
$$

Put $d_{n}=\alpha_{n} u+\left(1-\alpha_{n}\right) S t_{n}$. From $p=S p$ and (3.5), we have

$$
\begin{aligned}
\left\|d_{n}-p\right\| & \leq \alpha_{n}\|u-p\|+\left(1-\alpha_{n}\right)\left\|S t_{n}-S p\right\| \\
& \leq \alpha_{n}\|u-p\|+\left(1-\alpha_{n}\right)\left\|x_{n}-p\right\| .
\end{aligned}
$$


Thus, it follows from (3.6) that

$$
\begin{aligned}
\left\|x_{n+1}-p\right\| \leq & \beta_{n}\left\|x_{n}-p\right\|+\left(1-\beta_{n}\right)\left\|d_{n}-p\right\| \\
\leq & {\left[1-\alpha_{n}\left(1-\beta_{n}\right)\right]\left\|x_{n}-p\right\|+\alpha_{n}\left(1-\beta_{n}\right)\|u-p\| } \\
\leq & \max \left\{\left\|x_{n}-p\right\|,\|u-p\|\right\} \\
& \vdots \\
& \leq \max \left\{\left\|x_{0}-p\right\|,\|u-p\|\right\} .
\end{aligned}
$$

Hence, sequence $\left\{x_{n}\right\}$ is bounded. So, we deduce from (3.3)-(3.5) that sequences $\left\{y_{n}\right\},\left\{z_{n}\right\}$, and $\left\{t_{n}\right\}$ are also bounded.

Next, we show $\lim _{n \rightarrow \infty}\left\|x_{n+1}-x_{n}\right\|=0$. From Lemma 2.1, we have

$$
\begin{aligned}
\left\|y_{n+1}-y_{n}\right\| & =\left\|J_{\gamma_{n+1}^{A}}^{A} x_{n+1}-J_{\gamma_{n+1}^{A}}^{A}\left(\frac{\gamma_{n+1}^{A}}{\gamma_{n}^{A}} x_{n}+\left(1-\frac{\gamma_{n+1}^{A}}{\gamma_{n}^{A}}\right) J_{\gamma_{n}^{A}}^{A} x_{n}\right)\right\| \\
& \leq\left\|x_{n+1}-x_{n}\right\|+\frac{\left|\gamma_{n+1}^{A}-\gamma_{n}^{A}\right|}{\gamma_{n}^{A}}\left\|x_{n}-J_{\gamma_{n}^{A}}^{A} x_{n}\right\| \\
& \leq\left\|x_{n+1}-x_{n}\right\|+K_{1}\left|\gamma_{n+1}^{A}-\gamma_{n}^{A}\right|,
\end{aligned}
$$

where

$$
K_{1}=\frac{\sup _{n}\left\{\left\|x_{n}-J_{\gamma_{n}^{A}}^{A} x_{n}\right\|\right\}}{r}<\infty .
$$

Similarly, we also have

$$
\left\|z_{n+1}-z_{n}\right\| \leq\left\|T y_{n+1}-T y_{n}\right\|+K_{2} \mid \gamma_{n+1}^{B}-\gamma_{n}^{B} \|,
$$

where

$$
K_{2}=\frac{\sup _{n}\left\{\left\|T y_{n}-J_{\gamma_{n}^{B}}^{B}\left(T y_{n}\right)\right\|\right\}}{r}<\infty .
$$

From Lemma 2.2 iii), we have

$$
\begin{aligned}
\left\|t_{n+1}-t_{n}\right\|^{2}= & \left\|y_{n+1}-y_{n}\right\|^{2}+\delta^{2}\left\|T^{*}\left[\left(z_{n+1}-T y_{n+1}\right)-\left(z_{n}-T y_{n}\right)\right]\right\|^{2} \\
& +2 \delta\left\langle y_{n+1}-y_{n}, T^{*}\left[\left(z_{n+1}-T y_{n+1}\right)-\left(z_{n}-T y_{n}\right)\right]\right\rangle \\
\leq & \left\|y_{n+1}-y_{n}\right\|^{2}+\delta^{2}\|T\|^{2}\left\|\left(z_{n+1}-T y_{n+1}\right)-\left(z_{n}-T y_{n}\right)\right\|^{2} \\
& +2 \delta\left\langle T y_{n+1}-T y_{n},\left(z_{n+1}-T y_{n+1}\right)-\left(z_{n}-T y_{n}\right)\right\rangle \\
\leq & \left\|y_{n+1}-y_{n}\right\|^{2}-\delta\left(2-\delta\|T\|^{2}\right)\left\|\left(z_{n+1}-T y_{n+1}\right)-\left(z_{n}-T y_{n}\right)\right\|^{2} .
\end{aligned}
$$

It follows from $d_{n}=\alpha_{n} u+\left(1-\alpha_{n}\right) S t_{n}$ that

$$
\begin{aligned}
\left\|d_{n+1}-d_{n}\right\| & \leq\left|\alpha_{n+1}-\alpha_{n}\right|\|u\|+\left\|\left(1-\alpha_{n+1}\right) S t_{n+1}-\left(1-\alpha_{n}\right) S t_{n}\right\| \\
& \leq\left|\alpha_{n+1}-\alpha_{n}\right|\|u\|+\left|\alpha_{n+1}-\alpha_{n}\right|\left\|S t_{n+1}\right\|+\left(1-\alpha_{n}\right)\left\|t_{n+1}-t_{n}\right\| \\
& \leq\left\|t_{n+1}-t_{n}\right\|+K_{3}\left|\alpha_{n+1}-\alpha_{n}\right|,
\end{aligned}
$$

where $K_{3}=\|u\|+\sup _{n}\left\{\left\|S t_{n}\right\|\right\}<\infty$. From (3.7), (3.9) and (3.10), we obtain

$$
\left\|d_{n+1}-d_{n}\right\| \leq\left\|x_{n+1}-x_{n}\right\|+K_{1}\left|\gamma_{n+1}^{A}-\gamma_{n}^{A}\right|+K_{3}\left|\alpha_{n+1}-\alpha_{n}\right| .
$$


Thus, from the conditions C1) and C3), we have

$$
\limsup _{n \rightarrow \infty}\left(\left\|d_{n+1}-d_{n}\right\|-\left\|x_{n+1}-x_{n}\right\|\right) \leq 0
$$

So, it follows from Lemma 2.4 that

$$
\lim _{n \rightarrow \infty}\left\|x_{n}-d_{n}\right\|=0
$$

Hence, we have

$$
\left\|x_{n+1}-d_{n}\right\|=\beta_{n}\left\|x_{n}-d_{n}\right\| \rightarrow 0
$$

which together with (3.11) yields that

$$
\lim _{n \rightarrow \infty}\left\|x_{n+1}-x_{n}\right\|=0 .
$$

Next, we prove that the set of weak cluster points of the sequence $\left\{x_{n}\right\}$ is contained in $\Omega$. Indeed, we denote by $\omega\left(x_{n}\right)$ the set of weak cluster points of the sequence $\left\{x_{n}\right\}$ and suppose that $x^{*}$ is an arbitrarily in $\omega\left(x_{n}\right)$. Then there is a subsequence $\left\{x_{n_{k}}\right\}$ of $\left\{x_{n}\right\}$ such that $x_{n_{k}} \rightarrow x^{*}$. From the convexity of the function $\|\cdot\|^{2}$ on $H_{1}$ and (3.5), we have

$$
\begin{aligned}
\left\|x_{n+1}-p\right\|^{2} & \leq \beta_{n}\left\|x_{n}-p\right\|^{2}+\left(1-\beta_{n}\right)\left\|\alpha_{n} u+\left(1-\alpha_{n}\right) S t_{n}-p\right\|^{2} \\
& \leq \beta_{n}\left\|x_{n}-p\right\|^{2}+\left(1-\beta_{n}\right)\left[\alpha_{n}\|u-p\|^{2}+\left(1-\alpha_{n}\right)\left\|t_{n}-p\right\|^{2}\right] \\
& \leq\left\|x_{n}-p\right\|^{2}+\alpha_{n}\|u-p\|^{2}-\left\|x_{n}-J_{\gamma_{n}^{A}}^{A} x_{n}\right\|^{2}-\delta\left(2-\delta\|T\|^{2}\right)\left\|z_{n}-T y_{n}\right\|^{2} .
\end{aligned}
$$

Thus, we obtain that

$$
\begin{aligned}
& \left\|x_{n}-J_{\gamma_{n}^{A}}^{A} x_{n}\right\|^{2}+\delta\left(2-\delta\|T\|^{2}\right)\left\|z_{n}-T y_{n}\right\|^{2} \\
& \leq\left(\left\|x_{n}-p\right\|^{2}-\left\|x_{n+1}-p\right\|^{2}\right)+\alpha_{n}\|u-p\|^{2} \\
& \leq\left(\left\|x_{n}-p\right\|+\left\|x_{n+1}-p\right\|\right)\left\|x_{n+1}-x_{n}\right\|+\alpha_{n}\|u-p\|^{2} .
\end{aligned}
$$

It follows from $\left\|x_{n+1}-x_{n}\right\| \rightarrow 0$, the conditions C3) and C4) that

$$
\lim _{n \rightarrow \infty}\left\|x_{n}-J_{\gamma_{n}^{A}}^{A} x_{n}\right\|^{2}=\lim _{n \rightarrow \infty}\left\|z_{n}-T y_{n}\right\|^{2}=0 \text {. }
$$

So, we have

$$
\lim _{n \rightarrow \infty}\left\|x_{n}-J_{\gamma_{n}^{A}}^{A} x_{n}\right\|=\lim _{n \rightarrow \infty}\left\|J_{\gamma_{n}^{B}}^{B}\left(T y_{n}\right)-T y_{n}\right\|=\lim _{n \rightarrow \infty}\left\|t_{n}-y_{n}\right\|=0 .
$$

From Lemma 2.2 i), we get

$$
\lim _{n \rightarrow \infty}\left\|x_{n}-J_{r}^{A} x_{n}\right\|=\lim _{n \rightarrow \infty}\left\|J_{r}^{B}\left(T y_{n}\right)-T y_{n}\right\|=0 .
$$

Since $x_{n_{k}} \rightarrow x^{*}$ and $\lim _{n \rightarrow \infty}\left\|x_{n}-y_{n}\right\|=0$, one has $y_{n_{k}} \rightarrow x^{*}$. Since $T$ is bounded linear operator, $T y_{n_{k}} \rightarrow T x^{*}$. By use of Lemma 2.4, we obtain $x^{*} \in A^{-1} 0$, and $T x^{*} \in B^{-1} 0$, that is, $x^{*} \in A^{-1} 0 \cap$ $T^{-1}\left(B^{-1} 0\right)$.

Now, we show that $x^{*} \in \operatorname{Fix}(S)$. Note that

$$
\left\|x_{n}-t_{n}\right\| \rightarrow 0 \text {. }
$$

From the condition C3), the boundedness of $\left\{S t_{n}\right\}$ and $\left\|d_{n}-S t_{n}\right\|=\alpha_{n}\left\|u-S t_{n}\right\|$, we get

$$
\lim _{n \rightarrow \infty}\left\|d_{n}-S t_{n}\right\|=0,
$$


which together with (3.11) yields

$$
\lim _{n \rightarrow \infty}\left\|x_{n}-S t_{n}\right\|=0
$$

Thus, it follows from (3.14) and (3.16) that

$$
\lim _{n \rightarrow \infty}\left\|t_{n}-S t_{n}\right\|=0 .
$$

Since $x_{n_{k}} \rightarrow x^{*}$ and (3.14), we have $t_{n_{k}} \rightarrow x^{*}$. So, from Lemma 2.4 and (3.17), we obtain $x^{*} \in \operatorname{Fix}(S)$. Hence, $x^{*} \in \Omega$. Consequently, $\omega\left(x_{n}\right) \subseteq \Omega$.

Finally, we show that $x_{n} \rightarrow x^{\dagger}=P_{\Omega}^{H_{1}} u$. Putting $x^{\dagger}=P_{\Omega}^{H_{1}} u$, we have from (3.4) that

$$
\begin{aligned}
\left\|x_{n+1}-x^{\dagger}\right\|^{2}= & \beta_{n}\left\langle x_{n}-x^{\dagger}, x_{n+1}-x^{\dagger}\right\rangle+\left(1-\beta_{n}\right)\left\langle\alpha_{n} u+\left(1-\alpha_{n}\right) S t_{n}-x^{\dagger}, x_{n+1}-x^{\dagger}\right\rangle \\
\leq & \beta_{n} \frac{\left\|x_{n}-x^{\dagger}\right\|^{2}+\left\|x_{n+1}-x^{\dagger}\right\|^{2}}{2} \\
& +\left(1-\beta_{n}\right)\left(1-\alpha_{n}\right) \frac{\left\|t_{n}-x^{\dagger}\right\|^{2}+\left\|x_{n+1}-x^{\dagger}\right\|^{2}}{2} \\
& +\alpha_{n}\left(1-\beta_{n}\right)\left\langle u-x^{\dagger}, x_{n+1}-x^{\dagger}\right\rangle \\
\leq & \beta_{n} \frac{\left\|x_{n}-x^{\dagger}\right\|^{2}+\left\|x_{n+1}-x^{\dagger}\right\|^{2}}{2} \\
& +\left(1-\beta_{n}\right)\left(1-\alpha_{n}\right) \frac{\left\|x_{n}-x^{\dagger}\right\|^{2}+\left\|x_{n+1}-x^{\dagger}\right\|^{2}}{2} \\
& +\alpha_{n}\left(1-\beta_{n}\right)\left\langle u-x^{\dagger}, x_{n+1}-x^{\dagger}\right\rangle .
\end{aligned}
$$

Thus,

$$
\begin{aligned}
{\left[1+\alpha_{n}\left(1-\beta_{n}\right)\right]\left\|x_{n+1}-x^{\dagger}\right\|^{2} \leq } & {\left[1-\alpha_{n}\left(1-\beta_{n}\right)\right]\left\|x_{n}-x^{\dagger}\right\|^{2} } \\
& +2\left(1-\beta_{n}\right) \alpha_{n}\left\langle u-x^{\dagger}, x_{n+1}-x^{\dagger}\right\rangle .
\end{aligned}
$$

This implies that

$$
\begin{aligned}
\left\|x_{n+1}-x^{\dagger}\right\|^{2} \leq & {\left[1-\alpha_{n}\left(1-\beta_{n}\right)\right]\left\|x_{n}-x^{\dagger}\right\|^{2} } \\
& +\alpha_{n}\left(1-\beta_{n}\right) \frac{2}{1+\alpha_{n}\left(1-\beta_{n}\right)}\left\langle u-x^{\dagger}, x_{n+1}-x^{\dagger}\right\rangle .
\end{aligned}
$$

Let $s_{n}=\left\|x_{n}-x^{\dagger}\right\|^{2}$ and $c_{n}=\frac{2}{1+\alpha_{n}\left(1-\beta_{n}\right)}\left\langle u-x^{\dagger}, x_{n+1}-x^{\dagger}\right\rangle$. Then the inequality (3.18) can be rewritten in the following form

$$
s_{n+1} \leq\left[1-\alpha_{n}\left(1-\beta_{n}\right)\right] s_{n}+\alpha_{n}\left(1-\beta_{n}\right) c_{n} .
$$

Now, we will show that $\limsup _{n \rightarrow \infty} c_{n} \leq 0$. Indeed, suppose that $\left\{x_{n_{k}}\right\}$ is a subsequence of $\left\{x_{n}\right\}$ such that

$$
\limsup _{n \rightarrow \infty}\left\langle u-x^{\dagger}, x_{n}-x^{\dagger}\right\rangle=\lim _{k \rightarrow \infty}\left\langle u-x^{\dagger}, x_{n_{k}}-x^{\dagger}\right\rangle .
$$

Since $\left\{x_{n_{k}}\right\}$ is bounded, there exists a subsequence $\left\{x_{n_{k_{l}}}\right\}$ of $\left\{x_{n_{k}}\right\}$ such that $x_{n_{k_{l}}} \rightarrow x^{*}$. We may assume without loss of generality that $x_{n_{k}} \rightarrow x^{*}$. It follows from $\omega\left(x_{n}\right) \subseteq \Omega$ that $x^{*} \in \Omega$. So, from $x^{\dagger}=P_{\Omega}^{H_{1}} u$ and (2.2), we deduce that

$$
\limsup _{n \rightarrow \infty}\left\langle u-x^{\dagger}, x_{n}-x^{\dagger}\right\rangle=\left\langle u-x^{\dagger}, x^{*}-x^{\dagger}\right\rangle \leq 0,
$$


which together with (3.12), conditions C2), and C3) gets $\lim \sup _{n \rightarrow \infty} c_{n} \leq 0$. Since $\sum_{n=1}^{\infty} \alpha_{n}=$ $\infty$ and condition C2), we have $\sum_{n=1}^{\infty} \alpha_{n}\left(1-\beta_{n}\right)=\infty$. Hence, all conditions of Lemma 2.5 are satisfied. Therefore, we immediately deduce that $s_{n} \rightarrow 0$, that is, $x_{n} \rightarrow x^{\dagger}=P_{\Omega}^{H_{1}} u$. This completes the proof.

The viscosity approximation method, which was firstly introduced and investigated by Moudafi [15], acts as a bridge linking fixed point and variational inequality problem. Indeed, the fixed point of the potential nonlinear operator is also a unique solution to some monotone variational inequality.

Next, we give the following viscosity method

$$
\begin{aligned}
& u_{n}=J_{\gamma_{n}^{A}}^{A} e_{n}, \\
& v_{n}=J_{\gamma_{n}^{B}}^{B}\left(T u_{n}\right), \\
& w_{n}=u_{n}+\delta T^{*}\left(v_{n}-T u_{n}\right), \\
& e_{n+1}=\beta_{n} e_{n}+\left(1-\beta_{n}\right)\left[\alpha_{n} f\left(e_{n}\right)+\left(1-\alpha_{n}\right) S w_{n}\right], n \geq 0,
\end{aligned}
$$

where $f: H_{1} \rightarrow H_{1}$ is a contractive mapping from $H_{1}$ into itself with the contraction coefficient $c \in(0,1)$.

The convergence analysis of the above viscosity approximation method is easy to obtain with the aid the Theorem 3.1. However, we here still give the proof for the sake of the completeness.

Theorem 3.2. If conditions $\mathrm{C} 1)-\mathrm{C} 4)$ are satisfied, then the sequence $\left\{e_{n}\right\}$ generated by (3.20) converges strongly to $x^{*} \in \Omega$ which is the unique solution to the variational inequality

$$
\left\langle(I-f) x^{*}, y-x^{*}\right\rangle \geq 0, \forall y \in \Omega \text {. }
$$

Proof. Since $P_{S}^{H_{1}} f$ is contractive mapping, Banach contraction mapping principle guarantees that $P_{S}^{H_{1}} f$ has a unique fixed point $x^{*}$ which is the unique solution of the variational inequality

$$
\left\langle(I-f) x^{*}, y-x^{*}\right\rangle \geq 0, \forall y \in \Omega \text {. }
$$

From Theorem 3.1, replacing $u$ by $f\left(x^{*}\right)$ in (3.2), we have the sequence $\left\{x_{n}\right\}$ converging strongly to $P_{\Omega}^{H_{1}} f\left(x^{*}\right)=x^{*}$.

Now we only need to prove that $\left\|e_{n}-x_{n}\right\| \rightarrow 0$, as $n \rightarrow \infty$. Note that

$$
\left\|e_{n+1}-x_{n+1}\right\| \leq \beta_{n}\left\|e_{n}-x_{n}\right\|+\left(1-\beta_{n}\right)\left[\alpha_{n} c\left\|e_{n}-x^{*}\right\|+\left(1-\alpha_{n}\right)\left\|w_{n}-t_{n}\right\|\right] .
$$

By use of Lemma 2.2 iii), we have

$$
\begin{aligned}
\left\|w_{n}-t_{n}\right\|^{2}= & \left\|u_{n}-y_{n}\right\|^{2}+\delta^{2}\left\|T^{*}\left[\left(v_{n}-T u_{n}\right)-\left(z_{n}-T y_{n}\right)\right]\right\|^{2} \\
& +2 \delta\left\langle u_{n}-y_{n}, T^{*}\left[\left(v_{n}-T u_{n}\right)-\left(z_{n}-T y_{n}\right)\right]\right\rangle \\
\leq & \left\|u_{n}-y_{n}\right\|^{2}-\delta\left(2-\delta\|T\|^{2}\right)\left\|\left(v_{n}-T u_{n}\right)-\left(z_{n}-T y_{n}\right)\right\|^{2} \\
\leq & \left\|u_{n}-y_{n}\right\|^{2} .
\end{aligned}
$$

From the nonexpansiveness of $J_{\gamma_{n}^{A}}^{A}$, we have

$$
\left\|u_{n}-y_{n}\right\| \leq\left\|e_{n}-x_{n}\right\| .
$$


Thanks to (3.21)-(3.23), we obtain

$$
\begin{aligned}
\left\|e_{n+1}-x_{n+1}\right\| & \leq \beta_{n}\left\|e_{n}-x_{n}\right\|+\left(1-\beta_{n}\right)\left[\alpha_{n} c\left\|e_{n}-x^{*}\right\|+\left(1-\alpha_{n}\right)\left\|e_{n}-x_{n}\right\|\right] \\
& \leq\left[1-\alpha_{n}\left(1-\beta_{n}\right)\right]\left\|e_{n}-x_{n}\right\|+\alpha_{n}\left(1-\beta_{n}\right) c\left(\left\|e_{n}-x_{n}\right\|+\left\|x_{n}-x^{*}\right\|\right) \\
& =\left[1-\alpha_{n}\left(1-\beta_{n}\right)(1-c)\right]\left\|e_{n}-x_{n}\right\|+\alpha_{n}\left(1-\beta_{n}\right) c\left\|x_{n}-x^{*}\right\| .
\end{aligned}
$$

From Lemma 2.5, we get $\lim _{n \rightarrow \infty}\left\|e_{n}-x_{n}\right\|=0$. Thus, $\lim _{n \rightarrow \infty}\left\|e_{n}-x^{*}\right\|=0$, we obtain $\lim _{n \rightarrow \infty}\left\|e_{n}-x^{*}\right\|=0$. This completes the proof.

If the mapping $S$ in Theorem 3.2 is an identity mapping, then we have the following result.

Corollary 3.1. Let $H_{1}$ and $H_{2}$ be two real Hilbert spaces and let $f: H_{1} \rightarrow H_{1}$ be a contractive mapping on $H_{1}$. Let $A: H_{1} \rightarrow 2^{H_{1}}$ and $B: H_{2} \rightarrow 2^{H_{2}}$ be two maximal monotone operators on $H_{1}$ and $H_{2}$, respectively. Let $T: H_{1} \rightarrow H_{2}$ be bounded linear operator from $H_{1}$ onto $H_{2}$ such that $\Omega=A^{-1} 0 \cap T^{-1}\left(B^{-1} 0\right) \neq \emptyset$. If conditions $\left.\left.\mathrm{C} 1\right)-\mathrm{C} 4\right)$ are satisfied, then the sequence $\left\{e_{n}\right\}$ generated by the following iterative method: $e_{0} \in H_{1}$ and

$$
\begin{aligned}
& u_{n}=J_{\gamma_{n}^{A}}^{A} e_{n}, \\
& v_{n}=J_{\gamma_{n}^{B}}^{B}\left(T u_{n}\right), \\
& w_{n}=u_{n}+\delta T^{*}\left(v_{n}-T u_{n}\right), \\
& e_{n+1}=\beta_{n} e_{n}+\left(1-\beta_{n}\right)\left[\alpha_{n} f\left(e_{n}\right)+\left(1-\alpha_{n}\right) w_{n}\right], n \geq 0,
\end{aligned}
$$

converges strongly to $x^{*} \in \Omega$, which is a unique solution of the variational inequality

$$
\left\langle(I-f) x^{*}, y-x^{*}\right\rangle \geq 0, \forall y \in \Omega \text {. }
$$

If $H_{1}=H_{2}, A x=B x=0$ for all $x \in H_{1}$ and $\gamma_{n}^{A}=\gamma_{n}^{B}=1$ for all $n \geq 1$ in Theorem 3.2, we obtain the following result.

Corollary 3.2. Let $H_{1}$ be a real Hilbert space and let $f: H_{1} \rightarrow H_{1}$ be a contractive mapping on $H_{1}$. Let $S: H_{1} \rightarrow H_{1}$ be a nonexpansive mapping such that $\Omega=\operatorname{Fix}(S) \neq \emptyset$. If conditions $\mathrm{C} 2)-\mathrm{C} 3)$ are satisfied, then the sequence $\left\{e_{n}\right\}$ generated by the following iterative method; $e_{0} \in H_{1}$,

$$
e_{n+1}=\beta_{n} e_{n}+\left(1-\beta_{n}\right)\left[\alpha_{n} f\left(e_{n}\right)+\left(1-\alpha_{n}\right) S e_{n}\right], n \geq 0,
$$

converges strongly to $x^{*} \in \Omega$, which is a unique solution of the variational inequality

$$
\left\langle(I-f) x^{*}, y-x^{*}\right\rangle \geq 0, \forall y \in \Omega \text {. }
$$

\section{ApPLicATiOnS}

4.1. Split minimum point problem. Let $H$ be a real Hilbert space and let $g: H \rightarrow(-\infty, \infty]$ be a proper, lower semicontinuous and convex function. The subdifferential of $g$ is the multi-valued mapping $\partial g: H \rightarrow 2^{H}$, which is defined by

$$
\partial g(x)=\{z \in H: g(y)-g(x) \geq\langle y-x, z\rangle, \forall y \in H\}
$$

for all $x \in H$. We know that $\partial g$ is the maximal monotone operator [17] and $x_{0} \in \arg \min _{x \in H} g(x)$ if and only if $\partial g\left(x_{0}\right) \ni 0$.

We have the following result on the split minimum point problem in two real Hilbert spaces. 
Theorem 4.1. Let $H_{1}$ and $H_{2}$ be two real Hilbert spaces and let $f: H_{1} \rightarrow H_{1}$ be a contractive mapping on $H_{1}$. Let $f_{1}: H_{1} \rightarrow(-\infty, \infty], f_{2}: H_{2} \rightarrow(-\infty, \infty]$ be two proper, lower semicontinuous and convex functions and let $\mathrm{T}: \mathrm{H}_{1} \rightarrow \mathrm{H}_{2}$ be a bounded linear operator such that

$$
\Omega=\arg \min _{x \in H_{1}} f_{1}(x) \cap T^{-1}\left(\arg \min _{x \in H_{2}} f_{2}(x)\right) \neq \emptyset .
$$

If conditions $\mathrm{C} 1)-\mathrm{C} 4)$ are satisfied, then the sequence $\left\{e_{n}\right\}$ generated by $e_{0} \in H_{1}$ and

$$
\begin{aligned}
& u_{n}=\arg \min _{x \in H_{1}}\left\{f_{1}(x)+\frac{1}{2 \gamma_{n}^{A}}\left\|x-e_{n}\right\|^{2}\right\}, \\
& v_{n}=\arg \min _{y \in H_{2}}\left\{f_{2}(y)+\frac{1}{2 \gamma_{n}^{B}}\left\|y-T u_{n}\right\|^{2}\right\}, \\
& w_{n}=u_{n}+\delta T^{*}\left(v_{n}-T u_{n}\right), \\
& e_{n+1}=\beta_{n} e_{n}+\left(1-\beta_{n}\right)\left[\alpha_{n} f\left(e_{n}\right)+\left(1-\alpha_{n}\right) w_{n}\right], n \geq 0,
\end{aligned}
$$

converges strongly to $x^{*} \in \Omega$, which is a unique solution of the variational inequality $\langle(I-$ f) $\left.x^{*}, y-x^{*}\right\rangle \geq 0, \forall y \in \Omega$.

Proof. First, we have $\Omega=\left(\partial f_{1}\right)^{-1}(0) \cap T^{-1}\left(\left(\partial f_{2}\right)^{-1}(0)\right)$. Note that

$$
u_{n}=\arg \min _{x \in H_{1}}\left\{f_{1}(x)+\frac{1}{2 \gamma_{n}^{A}}\left\|x-e_{n}\right\|^{2}\right\}
$$

if and only if

$$
\partial f_{1}\left(u_{n}\right)+\frac{1}{\gamma_{n}^{A}}\left(u_{n}-e_{n}\right) \ni 0
$$

which implies that $u_{n}=J_{\gamma_{n}^{A}}^{A} e_{n}$, where $A=\partial f_{1}$. Similarly, we also have $v_{n}=J_{\gamma_{n}^{B}}^{B}\left(T u_{n}\right)$ with $B=\partial f_{2}$. Thus, by using Corollary 3.1 , we get the desired conclusion immediately.

4.2. Split feasibility problem. Let $C$ be a nonempty, closed and convex subset of a real Hilbert space $H$. Let $i_{C}$ be the indicator function of $C$, that is,

$$
i_{C}(x)=\left\{\begin{array}{l}
0, \text { if } x \in C, \\
\infty, \text { if } x \notin C .
\end{array}\right.
$$

It is easy to see that $i_{C}$ is the proper, semicontinuous and convex function and its subdifferential $\partial i_{C}$ is a maximal monotone operator. Observe taht

$$
\partial i_{C}(u)=N(u, C)=\{v \in H:\langle u-y, f\rangle \geq 0 \forall y \in C\},
$$

where $N(u, C)$ is the normal cone of $C$ at $u$.

We denote by $J_{r}$ the resolvent operator of $\partial i_{C}$ with $r>0$. Suppose $u=J_{r} x$ for $x \in H$, i.e.,

$$
\frac{x-u}{r} \in \partial i_{C}(u)=N(u, C) \text {. }
$$

Thus,

$$
\langle x-u, u-y\rangle \geq 0, \quad \forall y \in C .
$$

From (2.2), we get $u=P_{C}^{H} x$. In view of Corollary 3.1, we have the following result for solving the split feasibility problem in Hilbert spaces immediately. 
Theorem 4.2. Let $H_{1}$ and $H_{2}$ be two real Hilbert spaces and let $f: H_{1} \rightarrow H_{1}$ be a contractive mapping on $H_{1}$. Let $Q_{i}, i=1,2$ be two closed convex subsets of $H_{i}$, respectively. Let $T: H_{1} \rightarrow$ $H_{2}$ be a bounded linear operator such that $\Omega=Q_{1} \cap T^{-1}\left(Q_{2}\right) \neq \emptyset$. If conditions $\left.\left.\mathrm{C} 2\right)-\mathrm{C} 4\right)$ are satisfied, then the sequence $\left\{e_{n}\right\}$ generated by $e_{0} \in H_{1}$ and

$$
\begin{aligned}
& u_{n}=P_{Q_{1}}^{H_{1}} e_{n}, \\
& v_{n}=P_{Q_{2}}^{H_{2}}\left(T u_{n}\right), \\
& w_{n}=u_{n}+\delta T^{*}\left(v_{n}-T u_{n}\right), \\
& e_{n+1}=\beta_{n} e_{n}+\left(1-\beta_{n}\right)\left[\alpha_{n} f\left(e_{n}\right)+\left(1-\alpha_{n}\right) w_{n}\right], n \geq 0,
\end{aligned}
$$

converges strongly to $x^{*} \in \Omega$, which is a unique solution of the variational inequality $\langle(I-$ f) $\left.x^{*}, y-x^{*}\right\rangle \geq 0, \forall y \in \Omega$.

4.3. Split equilibrium problem. Let $C$ be a nonempty closed convex subset of a Hilbert space $H$. Let $F$ be a bifunction of $C \times C$ into $\mathbb{R}$, where $\mathbb{R}$ denotes the set of real numbers. Recall the following equilibrium problem:

$$
\text { find } x \in C \text { such that } F(x, y) \geq 0, \forall y \in C \text {. }
$$

To study the equilibrium problem, we may assume that $F$ satisfies the following restrictions:

(A1) $F(x, x)=0$ for all $x \in C$;

(A2) $F$ is monotone, i.e., $F(x, y)+F(y, x) \leq 0$ for all $x, y \in C$;

(A3) for each $x, y, z \in C, \lim _{t \downarrow 0} F(t z+(1-t) x, y) \leq F(x, y)$;

(A4) for each $x \in C, y \longmapsto F(x, y)$ is convex and lower semi-continuous.

We need the following lemma for solving the equilibrium problem.

Lemma 4.1. [21] Let $F$ be a bifunction from $C \times C$ to $\mathbb{R}$ which satisfies (A1)-(A4), and let $A_{F}$ be a multivalued mapping of $H$ into itself defined by

$$
A_{F} x=\left\{\begin{array}{l}
\{z \in H: F(x, y) \geq\langle y-x, z\rangle, \forall y \in C\}, x \in C \\
\emptyset, x \notin C .
\end{array}\right.
$$

Then $A_{F}$ is a maximal monotone operator with the domain $D\left(A_{F}\right) \subset C$, $E P(F)=A_{F}^{-1} 0$, where $E P(F)$ stands for the solution set of (4.3), and the resolvent $T_{r}=$ $\left(I+r A_{F}\right)^{-1}$ is defined by

$$
T_{r}^{F} x=\left\{z \in C: F(z, y)+\frac{1}{r}\langle y-z, z-x\rangle \geq 0, \forall y \in C\right\}, \forall x \in H .
$$

Now, we have the following result.

Theorem 4.3. Let $H_{1}$ and $H_{2}$ be two real Hilbert spaces and let $f: H_{1} \rightarrow H_{1}$ be a contractive mapping on $H_{1}$. Let $F_{i}: H_{i} \times H_{i} \rightarrow \mathbb{R}, i=1,2$ be two bifunctions which satisfy the conditions (A1)-(A4) and let $T: H_{1} \rightarrow H_{2}$ be a bounded linear operator such that $\Omega=$ 
$E P\left(F_{1}\right) \cap T^{-1}\left(E P\left(F_{2}\right)\right) \neq \emptyset$. If conditions $\left.\left.\mathrm{C} 1\right)-\mathrm{C} 4\right)$ are satisfied, then the sequence $\left\{e_{n}\right\}$ generated by $e_{0} \in H_{1}$ and

$$
\begin{aligned}
& u_{n}=T_{\gamma_{n}^{A}}^{F_{1}}\left(e_{n}\right), \\
& v_{n}=T_{\gamma_{n}^{B}}^{F_{2}}\left(T u_{n}\right), \\
& w_{n}=u_{n}+\delta T^{*}\left(v_{n}-T u_{n}\right), \\
& e_{n+1}=\beta_{n} e_{n}+\left(1-\beta_{n}\right)\left[\alpha_{n} f\left(e_{n}\right)+\left(1-\alpha_{n}\right) w_{n}\right], n \geq 0,
\end{aligned}
$$

converges strongly to $x^{*} \in \Omega$, which is a unique solution of the variational inequality $\langle(I-$ f) $\left.x^{*}, y-x^{*}\right\rangle \geq 0, \forall y \in \Omega$.

4.4. Split variational inequality problem. Let $H$ be a real Hilbert space and let $C$ be a closed convex subset of $H$. Let $A: C \rightarrow H$ be a single-valued monotone operator which is hemicontinuous. Then a point $u \in C$ is said to be a solution of the variational inequality involving operator $A$ if $\langle y-u, A u\rangle \geq 0$ holds for all $y \in C$. We denote by $\operatorname{VI}(C, A)$ the set of all solutions of the variational inequality.

Theorem 4.4. Let $H_{i}, i=1,2$, be two real Hilbert spaces and let $C_{i}, i=1,2$, be two closed convex subsets of $H_{i}$, respectively. Let $f: H_{1} \rightarrow H_{1}$ be a contractive mapping on $H_{1}$. Let $A_{i}: C_{i} \rightarrow H_{i}, i=1,2$, be two single-valued monotone and hemicontinuous operators, and let $T: H_{1} \rightarrow H_{2}$ be a bounded linear operator such that $\Omega=V I\left(C_{1}, A_{1}\right) \cap T^{-1}\left(V I\left(C_{2}, A_{2}\right)\right) \neq \emptyset$. If conditions $\mathrm{C} 1)-\mathrm{C} 4)$ are satisfied, then the sequence $\left\{e_{n}\right\}$ generated by $e_{0} \in H_{1}$ and

$$
\begin{aligned}
& u_{n}=V I\left(C_{1}, \gamma_{n}^{A_{1}} A_{1}+I^{H_{1}}-e_{n}\right), \\
& v_{n}=V I\left(C_{2}, \gamma_{n}^{A_{2}} A_{2}-I^{H_{2}}-T u_{n}\right), \\
& w_{n}=u_{n}+\delta T^{*}\left(v_{n}-T u_{n}\right), \\
& e_{n+1}=\beta_{n} e_{n}+\left(1-\beta_{n}\right)\left[\alpha_{n} f\left(e_{n}\right)+\left(1-\alpha_{n}\right) w_{n}\right], n \geq 0,
\end{aligned}
$$

converges strongly to $x^{*} \in \Omega$, which is a unique solution of the variational inequality $\langle(I-$ f) $\left.x^{*}, y-x^{*}\right\rangle \geq 0, \forall y \in \Omega$.

Proof. Define a mapping $T_{A_{1}} \subset H_{1} \times H_{1}$ by

$$
T_{A_{1}} x= \begin{cases}A_{1} x+N_{C_{1}}(x), & x \in C_{1} \\ \emptyset, & x \notin C_{1},\end{cases}
$$

where $N_{C_{1}}(x)=\left\{z \in H_{1}:\langle y-x, z\rangle \leq 0\right.$ for all $\left.y \in C_{1}\right\}$.

By Rockafellar [18], we know that the operator $T_{A_{1}}$ is maximal monotone, and $T_{A_{1}}^{-1}(0)=$ $\operatorname{VI}\left(C_{1}, A_{1}\right)$. Note that

if and only if

$$
u_{n}=V I\left(C_{1}, \gamma_{n}^{A_{1}} A_{1}+I^{H_{1}}-e_{n}\right)
$$

$$
\left\langle y-u_{n}, \gamma_{n}^{A_{1}} A_{1}\left(u_{n}\right)+u_{n}-e_{n}\right\rangle \geq 0
$$

for all $y \in C_{1}$, that is, $-\gamma_{n}^{A_{1}} A_{1}\left(u_{n}\right)-u_{n}+e_{n} \in \gamma_{n}^{A_{1}} N_{C_{1}}\left(u_{n}\right)$. This implies that $u_{n}=J_{\gamma_{n}^{A_{1}}}^{T_{A_{1}}} e_{n}$. Similarly, if we define a mapping $T_{A_{2}} \subset H_{2} \times H_{2}$ by

$$
T_{A_{2}} x= \begin{cases}A_{1} x+N_{C_{2}}(x), & x \in C_{2} \\ \emptyset, & x \notin C_{2},\end{cases}
$$


then $T_{A_{2}}$ is maximal monotone, $T_{A_{2}}^{-1}(0)=V I\left(C_{2}, A_{2}\right)$ and $v_{n}=J_{\gamma_{n}^{A_{2}}}^{T_{A_{2}}}\left(T u_{n}\right)$. From Corollary 3.1, we get the desired conclusion easily.

\section{NUMERICAL EXPERIMENTS}

The algorithms are implemented in MALAB 7.0 running on a HP Compaq 510, Core(TM) 2 Duo CPU. T5870 with $2.0 \mathrm{GHz}$ and 2GB RAM.

Example 5.1. Consider the problem of finding an element $x^{\dagger} \in \mathbb{R}^{4}$ such that

$$
x^{\dagger} \in \Omega=\arg \min _{x \in \mathbb{R}^{4}} f_{1}(x) \cap T^{-1}\left(\underset{x \in \mathbb{R}^{4}}{\arg \min } f_{2}(x)\right),
$$

where $f_{1}$ and $f_{2}$ are defined by

$$
f_{i}(x)=\left\langle A_{i} x, x\right\rangle+\left\langle B_{i}, x\right\rangle+C_{i}, i=1,2
$$

with

$$
\begin{gathered}
A_{1}=\left(\begin{array}{cccc}
1 & 1 & -1 & 0 \\
1 & 1 & -1 & 0 \\
-1 & -1 & 1 & 0 \\
0 & 0 & 0 & 0
\end{array}\right), A_{2}=\left(\begin{array}{llll}
1 & 1 & 0 & 1 \\
1 & 1 & 0 & 1 \\
0 & 0 & 0 & 1 \\
1 & 1 & 1 & 1
\end{array}\right), \\
B_{1}=\left(\begin{array}{llll}
-4 & -4 & 4 & 0
\end{array}\right), B_{2}=\left(\begin{array}{llll}
-4 & -4 & 0 & 4
\end{array}\right), C_{1}, C_{2} \text { are any constants, }
\end{gathered}
$$

and $T: \mathbb{R}^{4} \rightarrow \mathbb{R}^{4}$ is a bounded linear operator which is defined by

$$
T x=\left(\begin{array}{llll}
1 & 1 & 0 & 0 \\
2 & 0 & 4 & 0 \\
3 & 4 & 5 & 0 \\
0 & 0 & 0 & 0
\end{array}\right)\left(\begin{array}{l}
x_{1} \\
x_{2} \\
x_{3} \\
x_{4}
\end{array}\right)
$$

for all $x=\left(x_{1}, x_{2}, x_{3}, x_{4}\right) \in \mathbb{R}^{4}$.

Then, it is easy to show that $f_{1}$ and $f_{2}$ are two proper continuous convex functions on $\mathbb{R}^{4}$ and $x^{\dagger} \in S$ if and only if $2 A_{1} x^{\dagger}+B_{1}=0$ and $2 A_{2}\left(T x^{\dagger}\right)+B_{2}=0$. So, the set of solutions $\Omega$ in Example 5.1 is defined by

$$
S=\left\{\left(\frac{60}{23},-\frac{266}{161},-\frac{168}{161}, a\right): a \in \mathbb{R}\right\},
$$

and if $x_{0}=(2,-1,-2,4)$ then

$$
x^{\dagger}=P_{\Omega}^{\mathbb{R}^{4}}\left(x_{0}\right)=\left(\frac{60}{23},-\frac{266}{161},-\frac{168}{161}, 4\right) \approx(2.60869565,-1.65217391,-1.04347826,4) .
$$

In Theorem 4.1, let $f(x)=x_{0}=(2,-1,-2,4)$ for all $x \in \mathbb{R}^{4}, \gamma_{n}^{A_{1}}=\gamma_{n}^{A_{2}}=1, \alpha_{n}=1 / n$ and $\beta_{n}=1 / 2$ for all $n \geq 0$. Then, we have the following table of numerical results (Table 1 ).

The behavior of $\mathrm{TOL}_{n}$ in the case $\mathrm{TOL}_{n}<10^{-4}$ is described in Figure 1.

In Theorem 4.1 , let $f(x)=x / 5$ for all $x \in \mathbb{R}^{4}, \gamma_{n}^{A_{1}}=\gamma_{n}^{A_{2}}=1, \alpha_{n}=1 / n$ and $\beta_{n}=1 / 2$ for all $n \geq 0$. Then, we have the following table of numerical results (Table 1).

Note that, in these cases, at the $n$th iteration step, we define the function $\mathrm{TOL}_{n}$ by

$$
\mathrm{TOL}_{n}=\frac{1}{2}\left(\left\|2 A_{1} x_{n}+B_{1}\right\|^{2}+\left\|2 A_{2}\left(T x_{n}\right)+B_{2}\right\|^{2}\right)
$$




\begin{tabular}{clll}
\hline$\varepsilon$ & $n$ & TOL $_{n}$ & $x_{n}$ \\
\hline \hline $10^{-2}$ & 4460 & $9.99978481 \mathrm{e}-003$ & $(2.60154635,-1.64518849,-1.04377354,4)$ \\
$10^{-3}$ & 44450 & $9.99995419 \mathrm{e}-004$ & $(2.60798073,-1.65147533,-1.04350784,4)$ \\
$10^{-4}$ & 444351 & $9.99999830 \mathrm{e}-005$ & $(2.60862416,-1.65210405,-1.04348121,4)$ \\
\hline \multicolumn{4}{c}{ TABLE 1. Table of numerical results with TOL $_{n}=\left\|x_{n}-x^{\dagger}\right\|$}
\end{tabular}

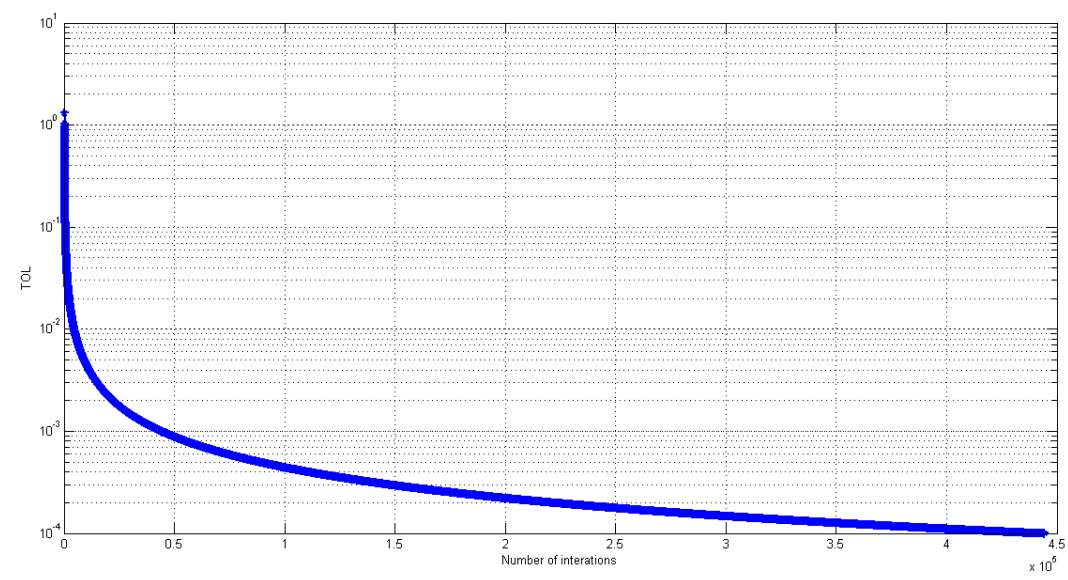

FIGURE 1. The behavior of $\mathrm{TOL}_{n}$ with the stop condition $\mathrm{TOL}_{n}<10^{-4}$

\begin{tabular}{clll}
\hline$\varepsilon$ & $n$ & TOL $_{n}$ & $x_{n}$ \\
\hline \hline $10^{-2}$ & 3945 & $9.995938 \mathrm{e}-003$ & $(2.586882,-1.631553,-1.043644,1.254370 \mathrm{e}-002)$ \\
$10^{-3}$ & 12415 & $9.999292 \mathrm{e}-004$ & $(2.601796,-1.645652,-1.043530,6.305143 \mathrm{e}-003)$ \\
$10^{-4}$ & 39202 & $9.999527 \mathrm{e}-005$ & $(2.606513,-1.650111,-1.043494,3.162867 \mathrm{e}-003)$ \\
$10^{-5}$ & 123908 & $9.999958 \mathrm{e}-006$ & $(2.608005,-1.651521,-1.043483,1.585649 \mathrm{e}-003)$ \\
$10^{-6}$ & 391774 & $9.999991 \mathrm{e}-007$ & $(2.608477,-1.651967,-1.043479,7.947777 \mathrm{e}-004)$ \\
\hline
\end{tabular}

TABLE 2. Table of numerical results

and use the condition $\mathrm{TOL}_{n}<\varepsilon$ to stop the iteration process, where $\varepsilon$ is an initial error.

The behavior of $\mathrm{TOL}_{n}$ in the case $\mathrm{TOL}_{n}<10^{-6}$ is described in Figure 2:

Remark 5.1. If, in Theorem $4.1, f(x)=x / 5$ for all $x \in \mathbb{R}^{3}$, then

$$
x^{\dagger}=P_{\Omega} 0 \approx(2.60869565,-1.65217391,-1.04347826,0) .
$$

Example 5.2. Consider the following problem: Find an element $x^{\dagger} \in \mathbb{R}^{40}$ such that

$$
x^{\dagger} \in \Omega=S_{1} \cap T^{-1}\left(S_{2}\right) \neq \emptyset,
$$

where $S_{1}=\left\{x \in \mathbb{R}^{40}:\left\|x-a_{1}\right\|^{2} \leq R_{1}^{2}\right\}, S_{2}=\left\{x \in \mathbb{R}^{50}:\left\|x-a_{2}\right\|^{2} \leq R_{2}^{2}\right\}$ and $T: \mathbb{R}^{40} \rightarrow \mathbb{R}^{50}$ is a bounded linear operator whose matrix has the elements are randomly generated in $[-10,10]$. 


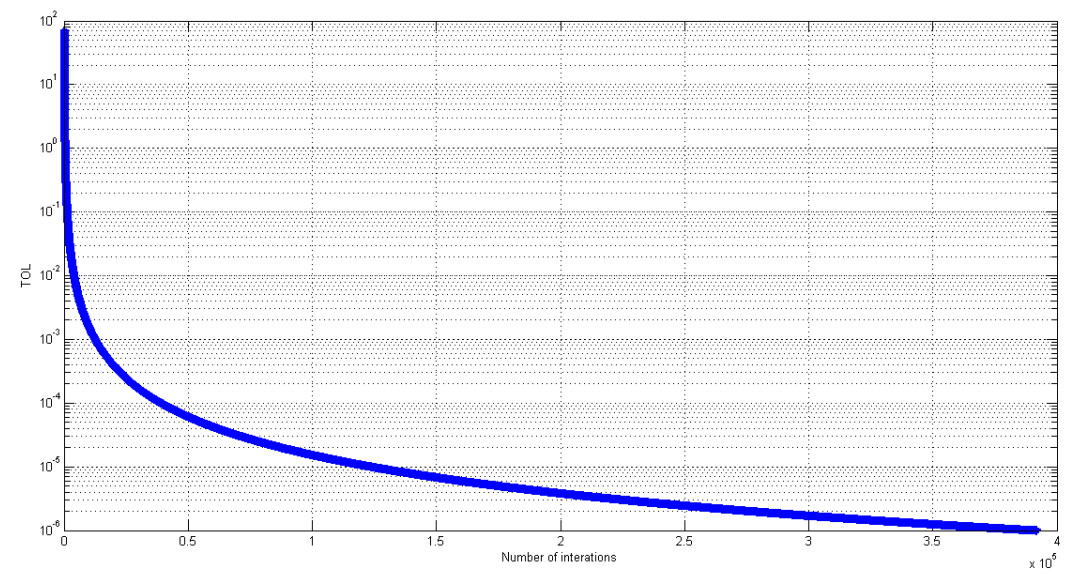

FIGURE 2. The behavior of $\mathrm{TOL}_{n}$ with the stop condition $\mathrm{TOL}_{n}<10^{-6}$

Now, we test the convergence of the sequence $\left\{x_{n}\right\}$ which is defined in Theorem 4.2 with the centers $a_{1}, a_{2}$ randomly generated in $[-1,1]$, the radius $R_{1}, R_{2}$ randomly generated in $[7,8]$ and $[9,10]$, respectively, and the initial $x_{0}$ randomly generated in $[-5,5]$.

It is easy to see that, in this case, $\Omega=S_{1} \cap T^{-1}\left(S_{2}\right) \neq \emptyset$ because $0 \in \Omega$.

Remark 5.2. In this example, the function TOL is defined by

$$
\mathrm{TOL}_{n}=\frac{1}{2}\left(\left\|x_{n}-P_{S_{1}}^{\mathbb{R}^{40}}\left(x_{n}\right)\right\|^{2}+\left\|T x_{n}-P_{S_{2}}^{\mathbb{R}^{50}}\left(T x_{n}\right)\right\|^{2}\right),
$$

for all $n \geq 1$. Note that, at the $n$th step, if $\mathrm{TOL}_{n}=0$ then $x_{n} \in S$, that is, $x_{n}$ is a solution of this problem.

After five attempts with the randomized data and $f(x)=x_{0}$ or $f(x)=x / 2$ for all $x \in \mathbb{R}^{40}$, $\alpha_{n}=1 / n, \beta_{n}=1 / 2$ for all $n \geq 1$ and $\delta=1 /\|T\|^{2}$, we obtain the following table of numerical results (Table 3 ).

The behavior of $\mathrm{TOL}_{n}$ in the Table 3 is described in Figure 3 and Figure 4.

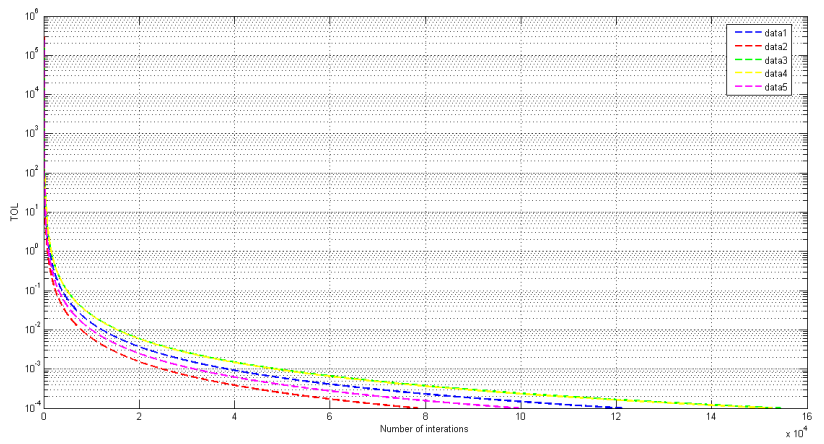

FIGURE 3. The behavior of $\mathrm{TOL}_{n}$ with the stop condition

$$
\mathrm{TOL}_{n}<10^{-4} \text { and } f(x)=x_{0}
$$




\begin{tabular}{|c|c|c|c|c|c|}
\hline \multicolumn{3}{|c|}{ Stop condition: $\mathrm{TOL}_{n}<10^{-3}$} & \multicolumn{3}{|c|}{ Stop condition: $\mathrm{TOL}_{n}<10^{-4}$} \\
\hline No. & $\mathrm{TOL}_{n}$ & $n$ & No. & $\mathrm{TOL}_{n}$ & $n$ \\
\hline \multicolumn{6}{|c|}{$f(x)=x_{0}$} \\
\hline 1 & $9.999930604862115 e-004$ & 38381 & 1 & $9.999978097851798 e-005$ & 121258 \\
\hline 2 & $9.999759540680524 e-004$ & 24794 & 2 & $9.999922131723621 e-005$ & 78401 \\
\hline 3 & $9.999842360618018 e-004$ & 48879 & 3 & $9.999944518918451 e-005$ & 154551 \\
\hline 4 & $9.999952749402601 e-004$ & 48303 & 4 & $9.999993798300586 e-005$ & 152641 \\
\hline 5 & $9.999536072386973 e-004$ & 31470 & 5 & $9.999836001196085 e-005$ & 99529 \\
\hline \multicolumn{6}{|c|}{$f(x)=x / 2$} \\
\hline 1 & $8.402767592710540 e-004$ & 116 & 1 & $5.484656397249216 e-005$ & 55 \\
\hline 2 & $5.372343063055929 e-004$ & 53 & 2 & $7.095432510606713 e-007$ & 97 \\
\hline 3 & $8.597287933614299 e-004$ & 60 & 3 & $3.859116638956402 e-005$ & 77 \\
\hline 4 & $4.419986354387474 e-004$ & 66 & 4 & 0 & 61 \\
\hline 5 & $2.435382620250198 e-004$ & 82 & 5 & $8.622270871144550 e-006$ & 74 \\
\hline
\end{tabular}

TABLE 3. Table of numerical results

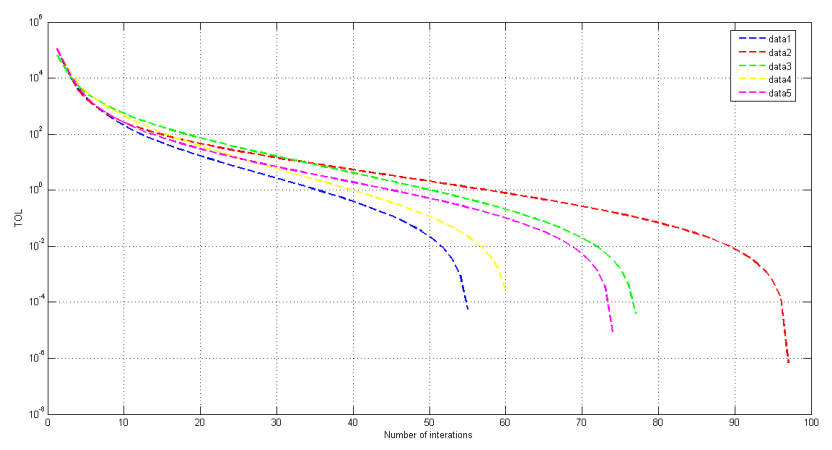

FIGURE 4. The behavior of $\mathrm{TOL}_{n}$ with the stop condition $\mathrm{TOL}_{n}<10^{-4}$ and $f(x)=x / 2$

\section{Acknowledgments}

The authors thank the referees for useful suggestions, which improved the presentation of this paper. The first author was supported by the Science and Technology Fund of Thai Nguyen University of Sciences.

\section{REFERENCES}

[1] V. Barbu, T. Precupanu, Convexity and Optimization in Banach spaces, Springer, Netherlands, 2012.

[2] E. Bonacker, A. Gibali, K.H. Küfer, Nesterov perturbations and projection methods applied to IMRT, J. Nonlinear Var. Anal. 4 (2019), 63-86.

[3] C. Byrne, Iterative oblique projection onto convex sets and the split feasibility problem, Inverse Probl. 18 (2002), 441-453.

[4] C. Byrne, A unified treatment of some iterative algorithms in signal processing and image reconstruction, Inverse Prob. 18 (2004), 103-120.

[5] Y. Censor, T. Elfving, A multi projection algorithm using Bregman projections in a product space, Numer. Algo. 8 (1994), 221-239. 
[6] V. Dadashi, Shrinking projection algorithms for the split common null point problem, Bull. Aust. Math. Soc. 99 (2017), 299-306.

[7] B. Eicke, Iteration methods for convexly constrained ill-posed problems in Hilbert space, Numer. Funct. Anal. Optim. 13 (1992), 423-429.

[8] B. Halpern, Fixed points of nonexpanding maps, Bull. Amer. Math. Soc. 73 (1967), 591-597.

[9] J.K. Kim, T.M. Tuyen, Alternating resolvent algorithms for finding a common zero of two accretive operator in Banach spaces, J. Korean Math. Soc. 54 (2017), 1905-1926.

[10] J.K. Kim, T.M. Tuyen, New iterative methods for finding a common zero of a finite family of monotone operators in Hilbert spaces, Bull Korean Math. Soc. 54 (2017), 1347-1359.

[11] L.S. Liu, Ishikawa and Mann iterative process with errors for nonlinear strongly accretive mappings in Banach spaces, J. Math. Anal. Appl. 194 (1995), 114-125.

[12] W. Mann, Mean value methods in iteration, Proc. Amer. Math. Soc. 4 (1953), 506-510.

[13] A. Moudafi, Weak convergence theorems for nonexpansive mappings and equilibrium problems, J. Nonlinear Convex Anal. 9 (2008), 37-43.

[14] A. Moudafi, Viscosity approximation methods for fixed-points problems, J. Math. Ana. Appl. 241 (2000), 46-55.

[15] A. Moudafi, The split common fixed point problem for demicontractive mappings, Inverse Probl. 26 (2010), 055007.

[16] X. Qin, J.C. Yao, A viscosity iterative method for a split feasibility problem, J. Nonlinear Convex Anal. 20 (2019), 1497-1506.

[17] R.T. Rockafellar, On the maximal monotonicity of subdifferential mappings, Pacific J. Math. 33 (1970), 209-216.

[18] R.T. Rockafellar, On the maximality of sums of nonlinear monotone operators, Trans. Amer. Math. Soc. 149 (1970), 75-88.

[19] T. Suzuki, Strong convergence theorems for infinite families of nonexpansive mappings in general Banach spaces, Fixed Point Theory Appl. 1 (2005), 103-123.

[20] S. Takahashi, W. Takahashi, Strong convergence theorem for a generalized equilibrium problem and a nonexpansive mapping in a Hilbert space, Nonlinear Anal. 69 (2008), 1025-1033.

[21] S. Takahashi, W. Takahashi, M. Toyoda, Strong convergence theorems for maximal monotone operators with nonlinear mappings in Hilbert spaces, J. Optim. Theory Appl. 147 (2010), 27-41.

[22] W. Takahashi, The split feasibility problem in Banach spaces, J. Nonlinear Convex Anal. 15 (2014), 13491355.

[23] W. Takahashi, The split feasibility problem and the shrinking projection method in Banach spaces, J. Nonlinear Convex Anal. 16 (2015), 1449-1459.

[24] S. Takahashi, W. Takahashi, The split common null point problem and the shrinking projection method in Banach spaces, Optimization, 65 (2016), 281-287.

[25] W. Takahashi, The split common null point problem in Banach spaces, Arch. Math. 104 (2015), $357-365$.

[26] Y.C. Tang, L.W. Liu, Several iterative algorithms for solving the split common fixed point problem of directed operators with applications, Optimization, 65 (2016), 53-65.

[27] T.M. Tuyen, A strong convergence theorem for the split common null point problem in Banach spaces, Appl. Math. Optim. 79 (2019), 207-227.

[28] H.K Xu, A variable Krasnosel'skii-Mann algorithm and the multiple-set split feasibility problem, Inverse Probl. 22 (2006), 2021-2034.

[29] H.K Xu, Iterative methods for the split feasibility problem in infinite dimensional Hilbert spaces, Inverse Probl. 26 (2010), 105018.

[30] H. Zhou, X. Qin, Fixed Points of Nonlinear Operators. Iterative Methods, De Gruyter, Berlin, 2020. 